\title{
Natural course of sleep-disordered breathing after acute myocardial infarction
}

\author{
Stefan Buchner*, , Tanja Greimel ${ }^{*,+}$, Andrea Hetzenecker ${ }^{\#}$, Andreas Luchner*, \\ Okka W. Hamer", Kurt Debl*, Florian Poschenrieder", Claudia Fellner", \\ Günter A.J. Riegger*, Michael Pfeifer*, ${ }^{*}$ and Michael Arzt*
}

ABSTRACT: The aim of this study was to test whether an improvement of left ventricular ejection fraction (EF) in the early phase after acute myocardial infarction is associated with a reduction of the severity of central and obstructive sleep apnoea.

40 consecutive patients with acute myocardial infarction underwent polysomnography and cardiovascular magnetic resonance imaging within 5 days and 12 weeks after the event to assess sleep apnoea and cardiac function. We stratified the sample in patients who improved their left ventricular EF within 12 weeks by $\geqslant 5 \%$ (improved EF group, $\Delta E F 9 \pm 1 \%, n=16$ ) and in those who did not (unchanged EF group, $\Delta E F-1 \pm 1 \%, n=24$ ).

Prevalence of sleep apnoea ( $\geqslant 15$ apnoea and hypopnoea events $\cdot h^{-1}$ ) within $\leqslant 5$ days after myocardial infarction was $55 \%$. Apnoea and hypopnoea events $\cdot h^{-1}$ were significantly more reduced in the improved EF group compared with the unchanged EF group $(-10 \pm 3$ versus $1 \pm 3$ events $\left.\cdot h^{-1} ; p=0.036\right)$. This reduction was based on a significant alleviation of obstructive events (-7 \pm 2 versus $4 \pm 3$ events $\left.\cdot h^{-1} ; p=0.009\right)$, while the reduction of central events was similar between groups $(p=0.906)$.

An improvement of cardiac function early after myocardial infarction is associated with an alleviation of sleep apnoea. This finding suggests that re-evaluation of treatment indication for sleep apnoea is needed when a change in cardiac function occurs.

KEYWORDS: Acute myocardial infarction, coronary artery disease, heart failure, sleep apnoea, ventilation

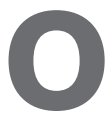
bstructive and central sleep apnoea (OSA and CSA) are highly prevalent in patients with chronic heart failure [1-3], as well as in patients with acute left ventricular failure, e.g. acute myocardial infarction (AMI) [4-6]. The interactions between sleep apnoea and heart failure (HF) are bi-directional. 1) Sleep apnoea may contribute to the progression of HF by exposing the heart to intermittent hypoxia, increased pre-load and afterload, sympathetic activation and vascular endothelial dysfunction. For example, in a previous observational study the presence of sleep apnoea early after AMI was linked to impaired recovery of cardiac function [6]. Ultimately, both OSA and CSA confer an increased mortality risk in patients with chronic HF [7-9]. 2) Conversely, there is epidemiological [10] and clinical evidence [11-15] that cardiovascular disease and especially $\mathrm{HF}$ can contribute to the development or worsening of sleep apnoea. It has been clearly demonstrated for several treatments of HF that improvement of cardiac function can alleviate or even abolish CSA. Examples of treatments improving cardiac function in patients with CSA include mitral valvuloplasty $[11,12]$, implantation of a biventricular cardiac device [13, 14] and cardiac transplantation [15]. However, the effect of an improvement of cardiac function on the severity of OSA has not been studied yet.

There is evidence from physiological experiments that fluid overload in HF with consecutive nocturnal rostral fluid shift is associated with upper airway narrowing [16] and the severity of OSA $[17,18]$. This fluid shift was directly related to the degree of leg oedema and sitting time, and inversely related to the degree of physical activity [17]. This mechanism may be reversed or attenuated by an improvement of cardiac function followed by less leg oedema and rostral fluid shift.

Thus, we hypothesised that an increase in ejection fraction (EF) in the early phase after AMI is associated with a reduction of the severity of CSA as well as OSA. To test this hypothesis, we studied a sample of patients with AMI and successful percutaneous coronary intervention (PCI). In this
AFFILIATIONS

*Klinik und Poliklinik für Innere Medizin II, Universitätsklinikum Regensburg,

"Institut für Röntgendiagnostik, Universitätsklinikum Regensburg, Regensburg, and

\#Zentrum für Pneumologie, Klinik Donaustauf, Donaustauf, Germany ${ }^{+}$Authors contributed equally.

CORRESPONDENCE

M. Arzt

Centre for Sleep Medicine, Dept of Internal Medicine ॥

University Hospital Regensburg Franz-Josef-Strauss-Allee 11

93053 Regensburg

Germany

E-mail: michael.arzt@klinik.uniregensburg.de

Received:

Oct 042011

Accepted after revision:

Feb 012012

First published online:

March 222012 
context, AMI is a model for acute HF, with one group of patients who improve their cardiac function and another whose left ventricular function remains impaired in the early phase after AMI when treated according to current international guidelines for therapy of AMI [19].

\section{METHODS}

\section{Patients}

In a prospective observational clinical study, patients with an AMI who were referred to the Universitätsklinikum Regensburg, Regensburg, Germany, between March 2009 and May 2010 were evaluated for eligibility (fig. 1). Inclusion criteria were: 1) aged between 18 and 80 yrs; 2) primary AMI with ST elevation in ECG; 3) successful PCI within $24 \mathrm{~h}$ after AMI; and 4) written informed consent. Medications were given according to international guidelines [19].

Exclusion criteria were: 1 ) aged $<18$ or $>80$ yrs; 2 ) former AMI with Q-waves in the ECG; 3) indication for a second PCI or surgical myocardial revascularisation; 4) cardiac shock (need of catecholamines); 5) implanted cardiac device or other

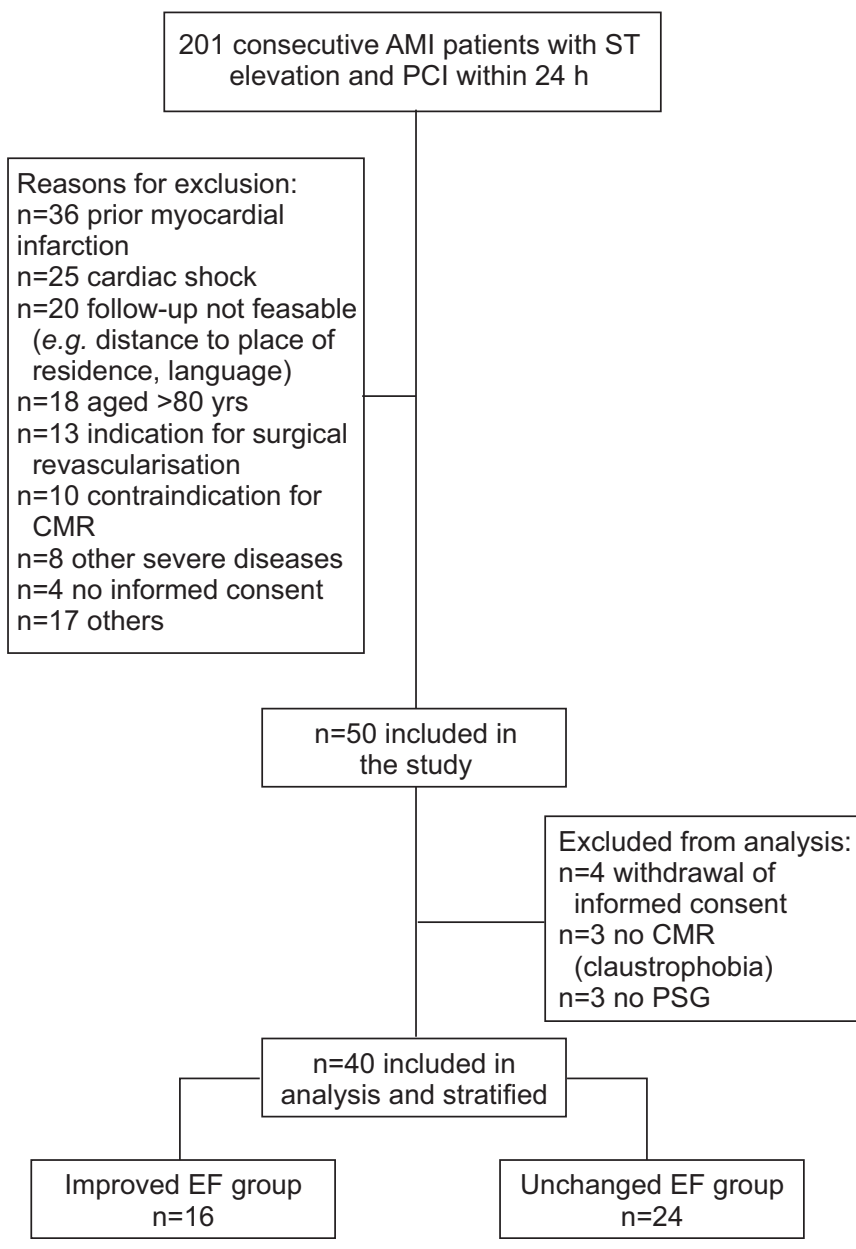

FIGURE 1. Flow diagram of patients included in the study. AMI: acute myocardial infarction; PCl: percutaneous coronary intervention; CMR: cardiovascular magnetic resonance; PSG: polysomnography; improved ejection fraction (EF) group: improvement of left ventricular EF within 12 weeks after AMl by $\geqslant 5 \%$; unchanged EF group: improvement of left ventricular EF within 12 weeks after AMI by $<5 \%$. contraindications for cardiovascular magnetic resonance imaging (CMR); 6) known treated or untreated sleep apnoea or at least moderate degree of lung diseases; and 7) former stroke. The protocol was approved by the local ethics committee and all patients provided written informed consent. Eligible patients underwent an overnight in-laboratory sleep study (polysomnography (PSG)) and CMR $\leqslant 5$ days and 12 weeks after PCI.

Patients were stratified according to the change of left ventricular $\mathrm{EF}(\Delta \mathrm{EF}=\mathrm{EF}$ at 12 weeks; $\mathrm{EF}$ baseline): 1) the sample of patients who improved their EF within 12 weeks after AMI by $\geqslant 5 \%$ (improved EF group); and 2) those who did not $(\Delta \mathrm{EF}<5 \%$; unchanged $\mathrm{EF}$ group; fig. 1). An increase of $\mathrm{EF}$ by $\geqslant 5 \%$ was a strong predictor of survival in HF patients [20].

\section{Polysomnography}

PSG was performed in all subjects using standard polysomnographic techniques (Alice System; Respironics, Pittsburgh, PA, USA) [8]. Respiratory efforts were measured with the use of respiratory inductance plethysmography and airflow by nasal pressure. Sleep stages and arousals, as well as apnoeas, hypopnoeas and respiratory effort-related arousals (RERA), were determined according to the American Academy of Sleep Medicine (AASM) guidelines by one experienced sleep technician blinded to the clinical data [21]. Hypopnoea definition A was used [21]. In addition, hypopnoeas were classified as obstructive if there was out-of-phase motion of the ribcage and abdomen, or if airflow limitation was present. In order to achieve optimal distinction between obstructive and central hypopnoeas without using an oesophageal balloon, we used additional criteria, such as flattening, snoring, paradoxical effort movements, arousal position relative to hypopnoeas and associated sleep stage (rapid eye movement (REM)/non-REM) [22]. CSA was defined as $>50 \%$ central apnoeas and hypopnoeas of all apnoeas and hypopnoeas.

\section{Cardiovascular magnetic resonance}

CMR studies were performed on a clinical 1.5 Tesla scanner (Avanto, Siemens Healthcare Sector, Erlangen, Germany) using a phased array receiver coil during breath-hold and that was ECG triggered. Examination of ventricular function was performed by acquisition of steady-state free precession (SSFP) cine images in standard short axis planes (trueFISP; slice thickness $8 \mathrm{~mm}$, inter-slice gap $2 \mathrm{~mm}$, repetition time $60.06 \mathrm{~ms}$, echo time $1.16 \mathrm{~ms}$, flip angle $60^{\circ}$, matrix size $134 \times 192$ and readout pixel bandwidth $930 \mathrm{~Hz} \cdot \mathrm{pixel}^{-1}$ ). The number of Fourier lines per heart beat was adjusted to allow the acquisition of 25 cardiac phases covering systole and diastole within a cardiac cycle. The field of view was $300 \mathrm{~mm}$ on average and was adapted to the size of the patient. Calculation of left ventricular volumes and EF was performed in the serial short axis slices using commercially available software (syngo Argus, version B15; Siemens Healthcare Sector).

\section{Statistical analysis}

Continuous data are expressed as mean $\pm \mathrm{SD}$, unless otherwise indicated. Differences between groups were assessed by twosided unpaired t-tests for continuous variables and by Chisquared tests for nominal variables. If the expected counts were $<5$, Fisher's exact test was used. ANCOVA was used to compare changes of outcome variables over time between groups. A twosided $\mathrm{p}$-value of $<0.05$ was considered as statistically significant. 
All analyses were performed using SPSS 18.0 (SPSS Inc., Chicago, IL, USA).

\section{RESULTS}

\section{Patient characteristics}

50 consecutive patients who were admitted to our institution with ST elevation AMI and underwent PCI within the first $24 \mathrm{~h}$ fulfilled the inclusion and exclusion criteria, and gave written informed consent (fig. 1). 10 of these patients were excluded from analysis due to withdrawal of informed consent, missing PSG or CMR data. None of the participants died or was lost to follow-up (fig. 1). The remaining 40 patients were stratified in the improved EF group $(n=16)$ and the unchanged EF group $(n=24$; fig. 1). The improved EF group showed a significant improvement in EF of $9 \pm 1 \%$, whereas the unchanged EF group remained unchanged $(-1 \pm 1 \%, \mathrm{p}<0.001$; fig. 2$)$. Compared with the patients in the improved EF group, the patients in the unchanged EF group showed no significant differences in age, sex, body mass index, weight or smoking status at baseline (table 1). Furthermore, maximum creatine kinase, systolic and diastolic blood pressure, heart rate, creatinine and EF at baseline were similar in both groups. Both groups showed no significant differences in pre-existing diseases such as diabetes, hypertension or coronary artery disease (table 1 ). N-terminal pro-brain natriuretic peptide (NT-proBNP) 1 day after the AMI was similarly elevated in the improved EF and the unchanged EF groups, respectively (table 1), indicating a moderate degree of HF $[23,24]$. Early after PCI, the majority of patients underwent chest radiographs and echocardiograms, which were not part of the study protocol; these indicated pulmonary congestion in $45 \%$ and a dilated vena cava inferior without respiratory variation $43 \%$, respectively. The frequency of such signs of pulmonary congestion were similar in both groups $(p=0.524$ and $\mathrm{p}=0.728$, respectively).

The mean Epworth Sleepiness Scale score at baseline was similar and in the normal range in both groups $(p=0.529$;

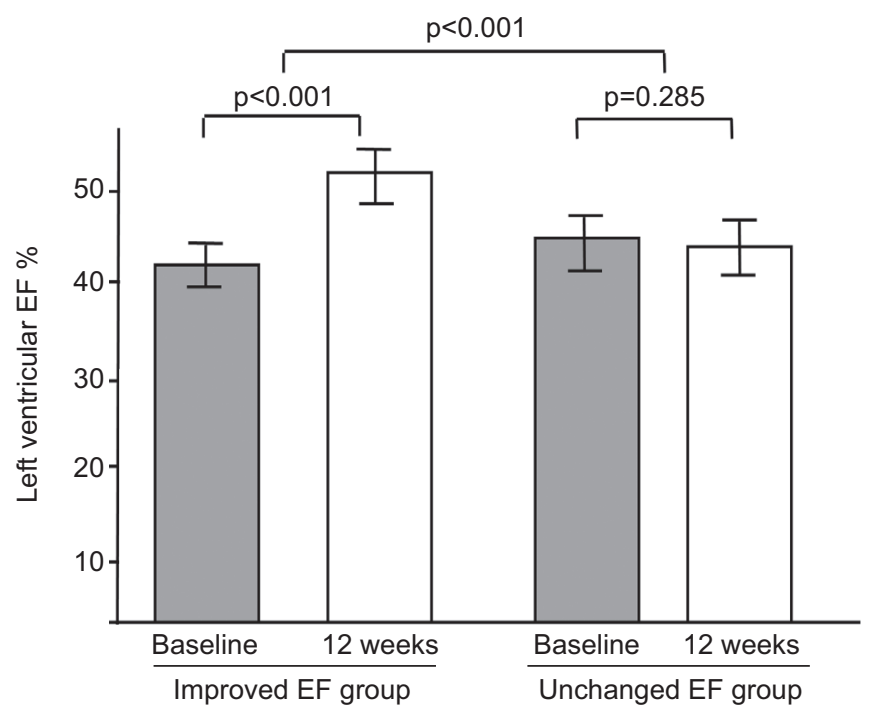

FIGURE 2. Change of left ventricular ejection fraction (EF). Improved EF group: improvement of left ventricular EF within 12 weeks after acute myocardial infarction (AMI) by $\geqslant 5 \%$; unchanged EF group: improvement of left ventricular EF within 12 weeks after AMl by $<5 \%$. table 1), indicating no excessive daytime sleepiness. There were no significant differences in the frequencies of $\beta$-blocker, angiotensin-converting enzyme inhibitors / AT1 antagonists or diuretic use at baseline and at follow-up time between the improved and unchanged EF groups (table 2).

\section{Type and severity of sleep apnoea in the early phase after AMI}

The prevalence of moderate-to-severe sleep apnoea (apnoea/ hypopnoea index $(\mathrm{AHI}) \geqslant 15$ events $\cdot \mathrm{h}^{-1}$ ) in the entire sample of AMI patients was $55 \%$ at $<5$ days after PCI, of whom half predominantly had CSA and half predominantly had OSA, respectively. After the 12-week observational period, there was a modest statistically nonsignificant reduction of sleep apnoea prevalence from $55 \%$ to $45 \%(\mathrm{p}=0.371)$.

To evaluate the change of sleep apnoea severity according to change in EF, we retrospectively stratified in individuals who improved their EF (the improved EF group) and in those who did not (the unchanged EF group; fig. 1). While the severity of sleep apnoea did not change significantly from $<5$ days to 12 weeks after PCI in the unchanged EF group (AHI $22 \pm 5$ to $23 \pm 5$ events $\left.\cdot h^{-1} ; p=0.756\right)$, AHI was significantly reduced in the improved EF group $\left(21 \pm 4\right.$ to $11 \pm 2$ events $\cdot h^{-1} ; p=0.010$; fig. 3). With respect to sleep apnoea type, the improved EF group showed a greater reduction of obstructive AHI compared with the unchanged EF group ( $p=0.009$; table 3 and fig. 3 ). In contrast, there was a modest nonsignificant decrease of central AHI that was similar in the improved EF and unchanged EF groups ( $p=0.906$; table 3 and fig. 3 ); this was paralleled by a similar rise of the awake arterialised capillary carbon dioxide tension ( $\mathrm{p}=0.958$; table 3 and fig. 4 ).

Such findings are confirmed in linear regression models using the changes of left ventricular EF and AHI, central AHI and obstructive AHI within the 12-week follow-up period as continuous variables. An increase of left ventricular EF was significantly associated with a decrease of $\mathrm{AHI}$ and obstructive AHI but not central AHI ( $\beta$ coefficient (95\% confidence interval): AHI 0.37 (0.17-1.69), $\mathrm{p}=0.018$; obstructive AHI 0.33 (0.01-1.25), $\mathrm{p}=0.045$; and central AHI $0.14(-0.35-0.95), \mathrm{p}=0.339)$.

While the prevalence of moderate-to-severe sleep apnoea $\left(\mathrm{AHI} \geqslant 15 \cdot \mathrm{h}^{-1}\right)$ was reduced from $69 \%$ to $38 \%(\mathrm{p}=0.077)$ in the improved EF group at baseline and at follow-up, the EF unchanged group's prevalence of moderate-to-severe sleep apnoea remained similar (from $46 \%$ to $50 \% ; p=0.773$ ). The prevalence of OSA was in the improved EF group (from $31 \%$ to $6 \%, \mathrm{p}=0.070)$, whereas it showed an increase in the unchanged EF group from baseline to follow-up time (from $25 \%$ to $31 \%$; $p=0.608$ ). In contrast to the EF unchanged group, $31 \%$ of the patients from the EF improved group shifted from a moderateto-severe degree of sleep apnoea to $<15$ apnoeas and hyperpnoeas per hour of sleep ( $0 \%$ versus $31 \%$; $\mathrm{p}=0.007)$.

The observed alleviation of sleep apnoea in the improved EF group was associated with a significant increase in mean arterial oxygen saturation, whereas minimum arterial oxygen saturation, sleep efficiency, and the proportion of N3 and rapid eye movement sleep stage did not change significantly (table 3). 


\begin{tabular}{|c|c|c|c|}
\hline & Improved left ventricular EF & Unchanged left ventricular EF & p-value \\
\hline Age yrs & $55 \pm 9$ & $56 \pm 11$ & 0.755 \\
\hline Males & $15(94)$ & $17(71)$ & 0.114 \\
\hline Weight kg & $87 \pm 11$ & $84 \pm 19$ & 0.672 \\
\hline Current smoking & $8(50)$ & $15(63)$ & 0.936 \\
\hline Maximal creatinine kinase $U \cdot L^{-1}$ & $1820 \pm 1471$ & $1930 \pm 1370$ & 0.813 \\
\hline Troponin I ng $\cdot \mathrm{mL}^{-1}$ & $27 \pm 81$ & $36 \pm 84$ & 0.739 \\
\hline NT-proBNP pg $\cdot \mathrm{mL}^{-1}$ & $1390 \pm 1180$ & $1693 \pm 1720$ & 0.546 \\
\hline Kreatinin $\mathrm{mg} \cdot \mathrm{dL}^{-1}$ & $1 \pm 0.4$ & $1 \pm 0.3$ & 0.216 \\
\hline Left ventricular EF \% & $43 \pm 2$ & $45 \pm 2$ & 0.421 \\
\hline Obstructive AHI events $\cdot h^{-1}$ & $12 \pm 10$ & $10 \pm 10$ & 0.526 \\
\hline
\end{tabular}

Data are presented as $\mathrm{n}(\%)$ or mean $\pm \mathrm{SD}$, unless otherwise stated. EF: ejection fraction; NT-proBNP: N-terminal probrain natriuretic peptide; AHI: apnoea/hypopnoea index; improved EF group: improvement of left ventricular EF within 12 weeks after acute myocardial infarction (AMI) by $\geqslant 5 \%$; unchanged $E F$ group: improvement of left ventricular EF within 12 weeks after AMI by $<5 \%$.

\section{Clinical course}

To evaluate whether either cardiac medication or fluid retention had an impact on the association between change of cardiac function and severity of sleep apnoea, we compared 1) the intake of cardiac medication at baseline and follow-up (table 1) and 2) the weight change, as a surrogate for fluid retention, over the follow-up period between the improved EF and the unchanged EF groups.

1) Patients of both the improved EF and the unchanged EF group showed no significant differences in medication at baseline and follow-up (table 1). All patients received acetylsalicylic acid and either clopidogrel or prasugrel for platelet aggregation inhibition and angiotensin-converting-enzyme (ACE)-inhibitors or angiotensin II type 1 (AT1) antagonists at baseline. The majority of patients from both groups received $\beta$-blockers, only one patient in the unchanged EF group did not receive a $\beta$-blocker at baseline, because of bradycardia. The vast majority of patients kept the ACE-inhibitors or AT1-antagonists and $\beta$-blockers until follow-up (table 2). All patients with clinical evidence of fluid overload (e.g. pulmonary or peripheral oedema) received diuretics. The use of loop diuretics and thiazide diuretics was similar in both groups at baseline and follow-up (table 2).

2) While the improved EF group showed a weight loss, the unchanged EF group gained weight (approximately $-1 \pm 1 \mathrm{~kg}$ versus $2 \pm 1 \mathrm{~kg} ; \mathrm{p}=0.062$ ).

\section{DISCUSSION}

This study provides several novel observations. First, 12 weeks following an AMI, patients have a very high prevalence of

\section{TABLE 2 Medication at baseline and follow-up}

\begin{tabular}{|c|c|c|c|}
\hline \multicolumn{4}{|l|}{ Baseline } \\
\hline$\beta$-blocker & $16(100)$ & $23(96)$ & 0.408 \\
\hline Loop diuretics & 2 (13) & $5(21)$ & 0.497 \\
\hline Thiazide diuretics & $3(19)$ & $4(17)$ & 0.865 \\
\hline$\beta$-blocker & $16(100)$ & $22(92)$ & 0.236 \\
\hline Loop diuretics & $2(13)$ & $6(26)$ & 0.301 \\
\hline Thiazide diuretics & 2 (13) & $5(22)$ & 0.460 \\
\hline
\end{tabular}

Data are presented as $n(\%)$, unless otherwise stated. EF: ejection fraction; ACE: angiotensin-converting-enzyme; AT1: angiotensin II type 1. 


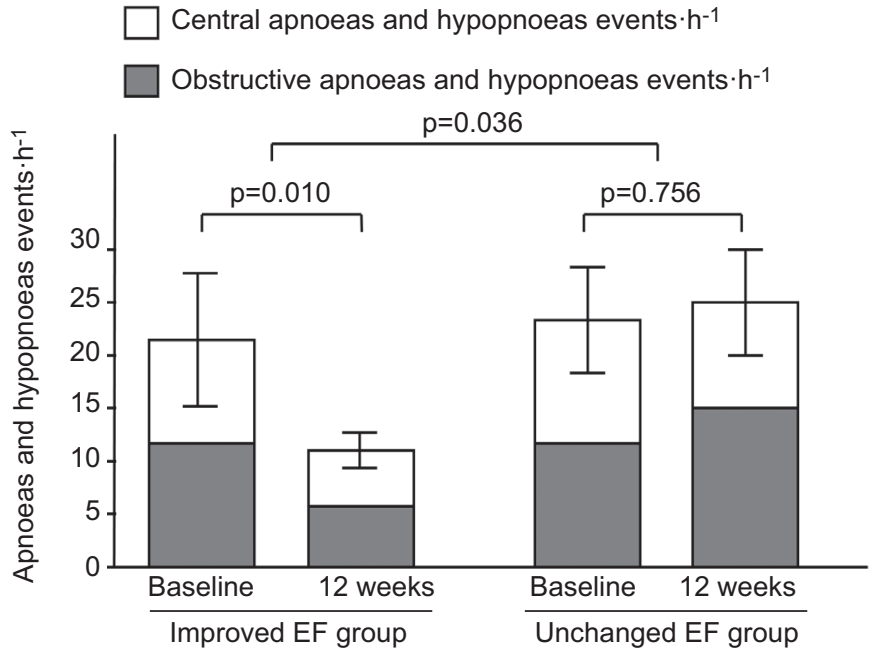

FIGURE 3. Change of the severity of sleep apnoea according to the change of cardiac function. EF: ejection fraction; improved EF group: improvement of left ventricular EF within 12 weeks after acute nyocardial infarction (AMI) by $\geqslant 5 \%$; unchanged EF group: improvement of left ventricular EF within 12 weeks after AMl by $<5 \%$.

moderate-to-severe sleep apnoea (45\%), of which $\sim 50 \%$ is obstructive and $50 \%$ is central in nature. Secondly, there is a modest reduction of sleep apnoea prevalence 12 weeks after a first AMI. Thirdly, improvement of cardiac function within the first 12 weeks after AMI is associated with a significant alleviation of sleep apnoea severity. In particular, especially obstructive apnoeas and hypopnoeas were reduced.

The prevalence of sleep apnoea in the acute phase after a first AMI in the current study is confirmed by several previous studies [5, 6, 25-27]. NAKASHIMA et al. [6] and YUMINO et al. [25] performed their sleep studies between 14-21 days after primary PCI, whereas other previous studies used a similar time-point to the present study to assess sleep apnoea at baseline ( $<5$ days after AMI) $[5,26,28]$.

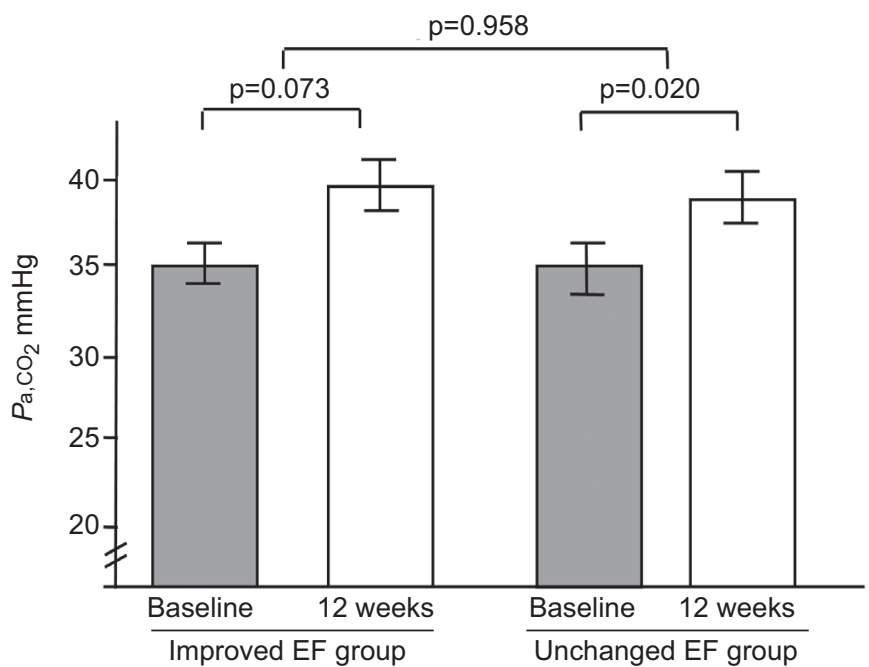

FIGURE 4. Change of arterial carbon dioxide tension $\left(\mathrm{Pa}_{1} \mathrm{CO}_{2}\right)$ according to the change of cardiac function. EF: ejection fraction; improved EF group: improvement of left ventricular EF within 12 weeks after acute nyocardial infarction (AMI) by $\geqslant 5 \%$; unchanged EF group: improvement of left ventricular EF within 12 weeks after AMl by $<5 \%$.

In the present study, the prevalence of an at least moderate degree of OSA and CSA was $28 \%$ and $28 \%$ within the first 5 days after AMI, respectively. In none of the patients was sleep apnoea suspected on clinical grounds, although $55 \%$ of patients had an at least moderate degree of sleep apnoea. One reason for this could be that, after AMI, patients did not report daytime hypersomnolence. This finding is in accordance with previous reports demonstrating a dissociation of sleep apnoea from hypersomnolence in patients with cardiovascular disease such as HF and stroke [29-31]. Furthermore, in the present study, both groups have poor sleep efficiency, similar to previous studies of patients with chronic HF [30].

PSG has been used in only one previous report studying sleep apnoea early after acute coronary syndrome [32], and the authors

TABLE 3 Respiratory and sleep characteristics according to the change of cardiac function

\begin{tabular}{|c|c|c|c|c|c|c|c|}
\hline & \multicolumn{3}{|c|}{ Improved left ventricular EF } & \multicolumn{3}{|c|}{ Unchanged left ventricular EF } & \multirow[t]{2}{*}{$\mathrm{p}$-value } \\
\hline & Baseline & 12 weeks & $\mathrm{p}$-value & Baseline & 12 weeks & $\mathrm{p}$-value & \\
\hline \multicolumn{8}{|l|}{ Respiratory characteristic } \\
\hline $\mathrm{AHI}$ events $\cdot \mathrm{h}^{-1}$ & $21 \pm 14$ & $11 \pm 9$ & 0.010 & $22 \pm 26$ & $23 \pm 24$ & 0.756 & 0.036 \\
\hline Obstructive $A H I$ events $\cdot h^{-1}$ & $12 \pm 10$ & $6 \pm 4$ & 0.008 & $10 \pm 10$ & $14 \pm 19$ & 0.166 & 0.009 \\
\hline Mean $\mathrm{Sa}, \mathrm{O}_{2} \%$ & $93.4 \pm 1.8$ & $94.4 \pm 1.4$ & 0.041 & $93.8 \pm 1.9$ & $94.0 \pm 1.5$ & 0.398 & 0.149 \\
\hline $\mathrm{Sa}, \mathrm{O}_{2}, \min$ & $85.0 \pm 7.9$ & $86.5 \pm 6.3$ & 0.546 & $85.6 \pm 6.3$ & $85.4 \pm 6.4$ & 0.335 & 0.471 \\
\hline \multicolumn{8}{|l|}{ Sleep characteristic } \\
\hline Sleep efficiency \% & $76 \pm 1$ & $74 \pm 1$ & 0.544 & $70 \pm 1$ & $70 \pm 1$ & 0.135 & 0.150 \\
\hline N3 $\%$ & $16 \pm 6$ & $14 \pm 6$ & 0.413 & $17 \pm 9$ & $16 \pm 10$ & 0.594 & 0.717 \\
\hline REM \% & $17 \pm 6$ & $15 \pm 7$ & 0.394 & $16 \pm 8$ & $14 \pm 7$ & 0.097 & 0.757 \\
\hline
\end{tabular}


made the distinction between OSAs and CSAs during sleep [21]. Similar to our data, moderate-to-severe OSA and CSA was prevalent in 45 and 37\%, respectively, of acute coronary syndrome patients [32]. However, the reported sample had less impaired cardiac function [32]. In contrast to our data, NAKASHIMA et al. [6] report a proportion of $43 \%$ OSA and no CSA in a sample of patients with AMI 14-21 days after the event. In this study [6], cardiac function was impaired similarly to our patients.

A significant proportion of CSA as observed in the present study, and a similar report from TAKAMA and KURABAYASHI [32], in a sample of AMI and acute coronary syndrome patients with a moderate degree of HF, is plausible. In our sample, left ventricular EF was impaired (EF 44\%). Thus the prevalence of CSA and OSA in the AMI patients of our study is comparable with that reported in samples of patients with chronic HF and with a similar degree of cardiac impairment $[1,3]$. In such samples of chronic HF, CSA, as well as OSA, is associated with impaired prognosis $[8,9]$.

None of the previous studies focused on the time course of sleep apnoea after the first AMI [6, 25, 27, 32, 33]. For the first time, we could demonstrate an $18 \%$ reduction of apnoea and hypopnoea events $\cdot \mathrm{h}^{-1}$ of sleep from $>5$ days to 12 weeks after an AMI in association with an improvement in cardiac function. The magnitude of this effect is of clinical relevance in the group of patients who improved their cardiac function because $31 \%$ of such patients shifted from a moderate-to-severe degree of sleep apnoea to mild or no sleep apnoea $(\mathrm{AHI}<15)$. While many experts would recommend treatment in patients with moderateto-severe sleep-disordered breathing (SDB) regardless of symptoms based on the potential effects on cardiovascular outcome, treatment is not indicated in those with mild SDB $(\mathrm{AHI}<15)$ without sleep apnoea-related symptoms [34]. Considering the high likelihood that the severity of sleep apnoea changes in association with changes of cardiac function, present data support the re-evaluation of sleep apnoea after improvement of cardiac function in order to prevent unjustified long-term treatment of patients without sleep apnoea or mild sleep apnoea without sleep apnoea-related symptoms. This finding is in line with previous studies that demonstrated that improvement of cardiac function by various therapies, such as implantation of a biventricular cardiac device [13, 14] and cardiac transplantation [15], correlates with an attenuation of the severity of sleep apnoea [11-15, 35]. In contrast to most studies [11-15], which observed patients with CSA and found an improvement in CSA, we demonstrated that, in our sample of AMI patients, the reduction of sleep apnoea in the group with an improvement of left ventricular EF is mainly caused by the reduction of OSA. There was a modest nonsignificant reduction of central respiratory events that was similar in patients with improved and unchanged cardiac function, respectively. This finding may be explained by the fact that the awake arterial carbon dioxide tension similarly increased in both groups within the 12-week follow-up period, suggesting an increase in ventilator control stability [36].

GARRIGUE et al. [35] observed a reduction in obstructive respiratory events as a consequence of increasing cardiac output by atrial overdrive pacing in patients with bradyarrythmias. However, this was not confirmed in a similar randomised controlled trial [37]. Thus, the current study complements previous reports by demonstrating that an improvement of cardiac function is also leading to a decrease in the severity of the OSA. We assume that the improvement of OSA in patients after AMI who improved their cardiac function correlates with 1) a decrease in hypervolaemia after the acute HF and 2) a reduction of ventilator control stability [38]. The observed loss of weight in the group of AMI patients who improved their left ventricular EF compared with those with stable ventricular function indicates that such patients reduced their fluid overload. As neither ventilator control stability nor neck circumference nor oedema in the neck were assessed, the present study design does not allow firm conclusions with respect to the underlying pathomechanisms for the reduction of obstructive respiratory events in conjunction with an improvement of cardiac function.

Findings have to be interpreted in the light of the following limitations. 1) The finding that the improved EF group lost more weight than the unchanged EF group would be in line with the potential pathomechanism that those patients who improved their EF may have less rostral fluid shift contributing to upper airway narrowing [16] and, hence, the severity of OSA [17, 18]. Other assessments to support this or other pathomechanisms (e.g. neck circumference and fluid displacement from the legs) were not performed. 2) In addition to cardiac function, other factors may have contributed to alleviation of OSA in the improved EF group. Our data support the fact that such effect was not related to time spent in the supine position and to changes in medication. 3) Breathing effort can be most accurately measured using an oesophageal balloon [21], which was not used in the present study. In spite of the implementation of additional criteria, such as flattening, snoring, paradoxical effort movements, arousal position relative to hypopnoeas and associated sleep stage (REM/non-REM), to distinguish between obstructive and central respiratory events [22], some misclassification may have biased the results with respect to the type of sleep apnoea.

In summary, we showed that moderate-to-severe sleep apnoea is present in $55 \%$ of patients in the early phase after AMI and that the number of patients with moderate-to-severe sleep apnoea is reduced by $18 \%$ after 12 weeks. Of those patients who improved their cardiac function within the first 12 weeks after AMI, a substantial proportion (31\%) shifted from moderate-to-severe sleep apnoea to no or mild sleep apnoea $\left(\mathrm{AHI}<15\right.$ events $\left.\cdot \mathrm{h}^{-1}\right)$ with no or uncertain indication for treatment [34]. In particular, obstructive apnoeas and hypopnoeas were reduced.

Thus, the present findings in patients in the early phase after AMI contribute to the clinically important information that the severity of sleep apnoea, including OSA, changes when an improvement in cardiac function occurs. Similar effects may be observed after several other medical or surgical interventions in patients with HF that improve cardiac function [11-15]. To prevent unjustified long-term treatment in patients with $\mathrm{HF}$, the present findings support the fact that the indication for treatment of sleep apnoea should be re-evaluated when an improvement in cardiac function occurs.

\section{STATEMENT OF INTEREST}

A statement of interest for M. Arzt can be found at www.erj. ersjournals.com/site/misc/statements.xhtml

\section{ACKNOWLEDGEMENTS}

The authors thank K. Reiter, D. Spanja, H. Koitsch and K. Ziczinski (Institut für Röntgendiagnostik, Universitätsklinik Regensburg, Regensburg, 
Germany), and A. Brandl-Novak, A. Braune and R. Luigart (Klinik und Poliklinik für Innere Medizin II, Universitätsklinik Regensburg) for excellent technical assistance.

\section{REFERENCES}

1 Oldenburg O, Lamp B, Faber L, et al. Sleep-disordered breathing in patients with symptomatic heart failure: a contemporary study of prevalence in and characteristics of 700 patients. Eur J Heart Fail 2007; 9: 251-257.

2 Schulz R, Blau A, Borgel J, et al. Sleep apnoea in heart failure. Eur Respir J 2007; 29: 1201-1205.

3 Sin DD, Fitzgerald F, Parker JD, et al. Risk factors for central and obstructive sleep apnea in 450 men and women with congestive heart failure. Am J Respir Crit Care Med 1999; 160: 1101-1106.

4 Hung J, Whitford EG, Parsons RW, et al. Association of sleep apnoea with myocardial infarction in men. Lancet 1990; 336: 261-264.

5 Lee $\mathrm{CH}$, Khoo SM, Tai BC, et al. Obstructive sleep apnea in patients admitted for acute myocardial infarction. Prevalence, predictors, and effect on microvascular perfusion. Chest 2009; 135: 1488-1495.

6 Nakashima H, Katayama T, Takagi C, et al. Obstructive sleep apnoea inhibits the recovery of left ventricular function in patients with acute myocardial infarction. Eur Heart J 2006; 27: 2317-2322.

7 Corra U, Pistono M, Mezzani A, et al. Sleep and exertional periodic breathing in chronic heart failure: prognostic importance and interdependence. Circulation 2006; 113: 44-50.

8 Jilek C, Krenn M, Sebah D, et al. Prognostic impact of sleep disordered breathing and its treatment in heart failure: an observational study. Eur J Heart Fail 2010; 13: 68-75.

9 Wang H, Parker JD, Newton GE, et al. Influence of obstructive sleep apnea on mortality in patients with heart failure. J Am Coll Cardiol 2007; 49: 1625-1631.

10 Chami HA, Resnick HE, Quan SF, et al. Association of incident cardiovascular disease with progression of sleep-disordered breathing. Circulation 2011; 123: 1280-1286.

11 Rubin AE, Gottlieb SH, Gold AR, et al. Elimination of central sleep apnoea by mitral valvuloplasty: the role of feedback delay in periodic breathing. Thorax 2004; 59: 174-176.

12 Yasuma F, Hayashi H, Noda S, et al. A case of mitral regurgitation whose nocturnal periodic breathing was improved after mitral valve replacement. Jpn Heart J 1995; 36: 267-272.

13 Sinha AM, Skobel EC, Breithardt OA, et al. Cardiac resynchronization therapy improves central sleep apnea and Cheyne-Stokes respiration in patients with chronic heart failure. J Am Coll Cardiol 2004; 44: 68-71.

14 Vazir A, Hastings PC, Morrell MJ, et al. Resolution of central sleep apnoea following implantation of a left ventricular assist device. Int J Cardiol 2010; 138: 317-319.

15 Mansfield DR, Solin P, Roebuck T, et al. The effect of successful heart transplant treatment of heart failure on central sleep apnea. Chest 2003; 124: 1675-1681.

16 Chiu KL, Ryan CM, Shiota S, et al. Fluid shift by lower body positive pressure increases pharyngeal resistance in healthy subjects. Am J Respir Crit Care Med 2006; 174: 1378-1383.

17 Yumino D, Redolfi S, Ruttanaumpawan P, et al. Nocturnal rostral fluid shift: a unifying concept for the pathogenesis of obstructive and central sleep apnea in men with heart failure. Circulation 2010; 121: 1598-1605.

18 Redolfi S, Yumino D, Ruttanaumpawan P, et al. Relationship between overnight rostral fluid shift and obstructive sleep apnea in nonobese men. Am J Respir Crit Care Med 2009; 179: 241-246.

19 Van de Werf F, Bax J, Betriu A, et al. Management of acute myocardial infarction in patients presenting with persistent ST-segment elevation: the Task Force on the Management of
ST-Segment Elevation Acute Myocardial Infarction of the European Society of Cardiology. Eur Heart J 2008; 29: 2909-2945.

20 Cintron G, Johnson G, Francis G, et al. Prognostic significance of serial changes in left ventricular ejection fraction in patients with congestive heart failure. The V-HeFT VA Cooperative Studies Group. Circulation 1993; 87: Suppl. 6, VI17-VI23.

21 Iber C, American Academy of Sleep Medicine. The AASM Manual for the Scoring of Sleep and Associated Events: Rules, Terminology and Technical Specifications. Westchester, American Academy of Sleep Medicine, 2007.

22 Randerath W, Morgenstem C, Oltmann A, et al. Evaluation of a new PSG based algorithm to differentiate obstructive and central hypopneas. Eur Respir J 2010; 36: Suppl. 54, p 4407.

23 Luchner A, Hengstenberg C, Lowel $\mathrm{H}$, et al. NT-ProBNP in outpatients after myocardial infarction: interaction between symptoms and left ventricular function and optimized cut-points. J Card Fail 2005; 11: S21-S27.

24 Buchner S, Debl K, Barlage S, et al. Dynamic changes in N-terminal pro-brain natriuretic peptide in acute coronary syndromes treated with percutaneous coronary intervention: a marker of ischemic burden., reperfusion and outcome. Clin Chem Lab Med 2010; 48: 875-881.

25 Yumino D, Tsurumi Y, Takagi A, et al. Impact of obstructive sleep apnea on clinical and angiographic outcomes following percutaneous coronary intervention in patients with acute coronary syndrome. Am J Cardiol 2007; 99: 26-30.

26 Mehra R, Principe-Rodriguez K, Kirchner HL, et al. Sleep apnea in acute coronary syndrome: high prevalence but low impact on 6month outcome. Sleep Med 2006; 7: 521-528.

27 Meng S, Fang L, Wang CQ, et al. Impact of obstructive sleep apnoea on clinical characteristics and outcomes in patients with acute coronary syndrome following percutaneous coronary intervention. J Int Med Res 2009; 37: 1343-1353.

28 Marin JM, Carrizo SJ, Kogan I. Obstructive sleep apnea and acute myocardial infarction: clinical implications of the association. Sleep 1998; 21: 809-815.

29 Arzt M, Harth M, Luchner A, et al. Enhanced ventilatory response to exercise in patients with chronic heart failure and central sleep apnea. Circulation 2003; 107: 1998-2003.

30 Arzt M, Young T, Finn L, et al. Sleepiness and sleep in patients with both systolic heart failure and obstructive sleep apnea. Arch Intern Med 2006; 166: 1716-1722.

31 Arzt M, Young T, Peppard PE, et al. Dissociation of obstructive sleep apnea from hypersomnolence and obesity in patients with stroke. Stroke 2010; 41: e129-134.

32 Takama N, Kurabayashi M. Possibility of close relationship between sleep disorder breathing and acute coronary syndrome. J Cardiol 2007; 49: 171-177.

33 Saito T, Yoshikawa T, Sakamoto Y, et al. Sleep apnea in patients with acute myocardial infarction. Crit Care Med 1991; 19: 938-941.

34 Mayer G, Fietze I, Fischer J, et al. S3-Leitlinie - Nicht erholsamer Schlaf/Schlafstörungen. Somnologie 2009; 13: 4-160.

35 Garrigue S, Bordier P, Jais P, et al. Benefit of atrial pacing in sleep apnea syndrome. N Engl J Med 2002; 346: 404-412.

36 Naughton M, Benard D, Tam A, et al. Role of hyperventilation in the pathogenesis of central sleep apneas in patients with congestive heart failure. Am Rev Respir Dis 1993; 148: 330-338.

37 Pepin JL, Defaye P, Garrigue S, et al. Overdrive atrial pacing does not improve obstructive sleep apnoea syndrome. Eur Respir J 2005; 25: 343-347.

38 Jordan AS, Wellman A, Edwards JK, et al. Respiratory control stability and upper airway collapsibility in men and women with obstructive sleep apnea. J Appl Physiol 2005; 99: 2020-2027. 